\title{
An Outer Bound for Multiple Access Channels with Correlated Sources
}

\author{
Wei Kang Sennur Ulukus \\ Department of Electrical and Computer Engineering \\ University of Maryland, College Park, MD 20742 \\ wkang@eng.umd.edu \\ ulukus@umd.edu
}

\begin{abstract}
The capacity region of the multiple access channel with correlated sources remains an open problem. Cover, EI Gamal and Salehi gave an achievable region in the form of singleletter entropy and mutual information expressions, without a single-letter converse. Cover, El Gamal and Salehi also suggested a converse in terms of some $n$-letter mutual informations, which are incomputable. We have proposed an upper bound for the sum rate of this channel in a single-letter expression, by utilizing a new necessary condition for the Markov chain constraint on the valid channel input distributions. In this paper, we extend our results from the sum rate to the entire capacity region. We obtain an outer bound for the capacity region of the multiple access channel with correlated sources in finite-letter expressions.
\end{abstract}

\section{INTRODUCTION}

The problem of determining the capacity region of the multiple access channel with correlated sources can be formulated as follows. Given a pair of correlated sources $(U, V)$ described by the joint probability distribution $p(u, v)$, and a discrete, memoryless, multiple access channel characterized by the transition probability $p\left(y \mid x_{1}, x_{2}\right)$, what are the necessary and sufficient conditions for the reliable transmission of $n$ independent identically distributed (i.i.d.) samples of the sources through the channel, in $n$ channel uses, as $n \rightarrow \infty$ ?

This problem was studied by Cover, El Gamal and Salehi in [1], in which an achievable region expressed by single-letter entropies and mutual informations was given as follows.

Theorem 1 ([1]) A source $(U, V)$ with joint distribution $p(u, v)$ can be sent with arbitrarily small probability of error over a multiple access channel characterized by $p\left(y \mid x_{1}, x_{2}\right)$, if there exist probability mass functions $p(s), p\left(x_{1} \mid u, s\right)$, $p\left(x_{2} \mid v, s\right)$, such that

$$
\begin{aligned}
H(U \mid V) & <I\left(X_{1} ; Y \mid X_{2}, V, S\right) \\
H(V \mid U) & <I\left(X_{2} ; Y \mid X_{1}, U, S\right) \\
H(U, V \mid W) & <I\left(X_{1}, X_{2} ; Y \mid W, S\right) \\
H(U, V) & <I\left(X_{1}, X_{2} ; Y\right)
\end{aligned}
$$

where

$$
\begin{aligned}
& p\left(s, u, v, x_{1}, x_{2}, y\right) \\
& \quad=p(s) p(u, v) p\left(x_{1} \mid u, s\right) p\left(x_{2} \mid v, s\right) p\left(y \mid x_{1}, x_{2}\right)
\end{aligned}
$$

This work was supported by NSF Grants CCR 03-11311, CCF 04-47613 and CCF 05-14846; and ARL/CTA Grant DAAD 19-01-2-0011. and

$$
w=f(u)=g(v)
$$

is the common information in the sense of Witsenhausen, Gacs and Korner (see [2]).

The above region can be simplified if there is no common information between $U$ and $V$ as follows [1]

$$
\begin{aligned}
& H(U \mid V)<I\left(X_{1} ; Y \mid X_{2}, V\right) \\
& H(V \mid U)<I\left(X_{2} ; Y \mid X_{1}, U\right) \\
& H(U, V)<I\left(X_{1}, X_{2} ; Y\right)
\end{aligned}
$$

where

$$
p\left(u, v, x_{1}, x_{2}, y\right)=p(u, v) p\left(x_{1} \mid u\right) p\left(x_{2} \mid v\right) p\left(y \mid x_{1}, x_{2}\right)
$$

This achievable region was shown to be suboptimal by Dueck [3].

Cover, El Gamal and Salehi [1] also provided a capacity result with both achievability and converse in incomputable expressions in the form of some $n$-letter mutual informations. Their result is restated in the following theorem.

Theorem 2 ([1]) The correlated sources $(U, V)$ can be communicated reliably over the discrete memoryless multiple access channel $p\left(y \mid x_{1}, x_{2}\right)$ if and only if

$$
[H(U \mid V), H(V \mid U), H(U, V)] \in \bigcup_{n=1}^{\infty} \mathcal{C}_{n}
$$

where

$$
\mathcal{C}_{n}=\left\{\left[R_{1}, R_{2}, R_{3}\right]:\left\{\begin{array}{l}
R_{1}<\frac{1}{n} I\left(X_{1}^{n} ; Y^{n} \mid X_{2}^{n}, V^{n}\right) \\
R_{2}<\frac{1}{n} I\left(X_{2}^{n} ; Y^{n} \mid X_{1}^{n}, U^{n}\right) \\
R_{3}<\frac{1}{n} I\left(X_{1}^{n}, X_{2}^{n} ; Y^{n}\right)
\end{array}\right\}\right.
$$

for some

$$
\begin{aligned}
& p\left(u^{n}, v^{n}, x_{1}^{n}, x_{2}^{n}, y^{n}\right)= \\
& \quad p\left(x_{1}^{n} \mid u^{n}\right) p\left(x_{2}^{n} \mid v^{n}\right) \prod_{i=1}^{n} p\left(u_{i}, v_{i}\right) \prod_{i=1}^{n} p\left(y_{i} \mid x_{1 i}, x_{2 i}\right)
\end{aligned}
$$

i.e., for some $X_{1}^{n}$ and $X_{2}^{n}$ that satisfy the Markov chain $X_{1}^{n} \rightarrow$ $U^{n} \rightarrow V^{n} \rightarrow X_{2}^{n}$.

In [4], we proposed an upper bound for the sum rate of this channel in a single-letter expression by utilizing a new 
necessary condition for the Markov chain constraint on the possible input distributions given in (13). In this paper, we extend our work in [4] from sum rate point to the entire capacity region, i.e., we derive a finite-letter outer bound for the capacity region of the multiple access channel with correlated sources.

\section{A New OUter Bound}

We propose a new outer bound as follows.

Theorem 3 If a pair of i.i.d. sources $(U, V)$ with joint distribution $p(u, v)$ can be transmitted reliably through a discrete, memoryless, multiple access channel characterized by $p\left(y \mid x_{1}, x_{2}\right)$, then

$$
\begin{aligned}
& H(U \mid V) \leq I\left(X_{1} ; Y \mid X_{2}, V^{m}, Q\right) \\
& H(V \mid U) \leq I\left(X_{2} ; Y \mid X_{1}, U^{m}, Q\right) \\
& H(U, V) \leq I\left(X_{1}, X_{2} ; Y \mid Q\right)
\end{aligned}
$$

for some positive integer $n$, and random variables $X_{1}, X_{2}$ and $Q$, such that

$$
\begin{aligned}
& p\left(x_{1}, x_{2}, y, u^{n}, v^{n}, q\right) \\
& \quad=p(q) p\left(x_{1} \mid u^{n}, q\right) p\left(x_{2} \mid v^{n}, q\right) p\left(y \mid x_{1}, x_{2}\right) \prod_{i=1}^{n} p\left(u_{i}, v_{i}\right)
\end{aligned}
$$

where $\left(U^{n}, V^{n}\right)$ are $n$ samples of the i.i.d. sources, $\left(U^{m}, V^{m}\right)$ are the first $m$ samples of the sources, and $m$ is a non-negative finite integer.

Proof: Consider a given block code of length $n$ with the encoders $f_{1}: \mathcal{U}^{n} \longmapsto \mathcal{X}_{1}^{n}$ and $f_{2}: \mathcal{V}^{n} \longmapsto \mathcal{X}_{2}^{n}$ and decoder $g: \mathcal{Y}^{n} \longmapsto \mathcal{U}^{n} \times \mathcal{V}^{n}$. From Fano's inequality [5, p. 39], we have

$$
H\left(U^{n}, V^{n} \mid Y^{n}\right) \leq n \log _{2}|\mathcal{U} \times \mathcal{V}| P_{e}+1 \triangleq n \epsilon_{n}
$$

Since $m$ is a finite non-negative integer, we have $m<n$ when $n \rightarrow \infty$. We define $\left[\mathbf{U}_{i}^{T}, \mathbf{V}_{i}^{T}\right]^{T}$ as follows

$$
\left[\begin{array}{c}
\mathbf{U}_{i} \\
\mathbf{V}_{i}
\end{array}\right] \triangleq\left[\begin{array}{llll}
U_{i_{1}} & U_{i_{2}} & \cdots & U_{i_{m}} \\
V_{i_{1}} & V_{i_{2}} & \cdots & V_{i_{m}}
\end{array}\right]
$$

where $\left(U_{i_{j}}, V_{i_{j}}\right)$ is an arbitrary sample among the $n$-letter sources $\left(U^{n}, V^{n}\right)$, for $i=1, \ldots, n$ and $j=1, \ldots, m$. If $m=0,\left(\mathbf{U}_{i}, \mathbf{V}_{i}\right) \triangleq \emptyset$.
For a code, for which $P_{e} \rightarrow 0$, as $n \rightarrow \infty$, we have $\epsilon_{n} \rightarrow 0$. Then,

$$
\begin{array}{rl}
n & H(U \mid V)=H\left(U^{n} \mid V^{n}\right) \\
& =I\left(U^{n} ; Y^{n} \mid V^{n}\right)+H\left(U^{n} \mid Y^{n}, V^{n}\right) \\
& \leq I\left(U^{n} ; Y^{n} \mid V^{n}\right)+H\left(U^{n}, V^{n} \mid Y^{n}\right) \\
& \text { 1) } \\
& \leq I\left(U^{n} ; Y^{n} \mid V^{n}\right)+n \epsilon_{n} \\
& =H\left(Y^{n} \mid V^{n}\right)-H\left(Y^{n} \mid U^{n}, V^{n}\right)+n \epsilon_{n} \\
& \stackrel{2}{=} H\left(Y^{n} \mid X_{2}^{n}, V^{n}\right)-H\left(Y^{n} \mid X_{1}^{n}, X_{2}^{n}, U^{n}, V^{n}\right)+n \epsilon_{n} \\
& \stackrel{3}{=} H\left(Y^{n} \mid X_{2}^{n}, V^{n}\right)-H\left(Y^{n} \mid X_{1}^{n}, X_{2}^{n}\right)+n \epsilon_{n} \\
& \stackrel{4)}{=} \sum_{i=1}^{n}\left[H\left(Y_{i} \mid X_{2}^{n}, V^{n}, Y^{i-1}\right)-H\left(Y_{i} \mid X_{1 i}, X_{2 i}\right)\right]+n \epsilon_{n} \\
& \text { 5) } \\
\quad & \sum_{i=1}^{n}\left[H\left(Y_{i} \mid X_{2 i}, \mathbf{V}_{i}\right)-H\left(Y_{i} \mid X_{1 i}, X_{2 i}\right)\right]+n \epsilon_{n} \\
& \stackrel{6)}{=} \sum_{i=1}^{n}\left[H\left(Y_{i} \mid X_{2 i}, \mathbf{V}_{i}\right)-H\left(Y_{i} \mid X_{1 i}, X_{2 i}, \mathbf{V}_{i}\right)\right]+n \epsilon_{n} \\
& =\sum_{i=1}^{n} I\left(X_{1 i} ; Y_{i} \mid X_{2 i}, \mathbf{V}_{i}\right)+n \epsilon_{n}
\end{array}
$$

where

1) from Fano's inequality in (18);

2) from the fact that $X_{1}^{n}$ is the deterministic function of $U^{n}$ and $X_{2}^{n}$ is the deterministic function of $V^{n}$;

3) from $p\left(y^{n} \mid x_{1}^{n}, x_{2}^{n}, u^{n}, v^{n}\right)=p\left(y^{n} \mid x_{1}^{n}, x_{2}^{n}\right)$;

4) from the chain rule and the memoryless nature of the channel;

5) from the property that conditioning reduces entropy;

6) from $p\left(y_{i} \mid x_{1 i}, x_{2 i}, v_{i}^{m}\right)=p\left(y_{i} \mid x_{1 i}, x_{2 i}\right)$.

Using a symmetrical argument, we obtain

$$
n H(V \mid U) \leq \sum_{i=1}^{n} I\left(X_{2 i} ; Y_{i} \mid X_{1 i}, \mathbf{U}_{i}\right)+n \epsilon_{n}
$$

Moreover,

$$
\begin{array}{rl}
n & H(U, V)=H\left(U^{n}, V^{n}\right) \\
& =I\left(U^{n}, V^{n} ; Y^{n}\right)+H\left(U^{n}, V^{n} \mid Y^{n}\right) \\
& \leq I\left(U^{n}, V^{n} ; Y^{n}\right)+n \epsilon_{n} \\
& \leq I\left(X_{1}^{n}, X_{2}^{n} ; Y^{n}\right)+n \epsilon_{n} \\
& =H\left(Y^{n}\right)-H\left(Y^{n} \mid X_{1}^{n}, X_{2}^{n}\right)+n \epsilon_{n} \\
& =\sum_{i=1}^{n}\left[H\left(Y_{i} \mid Y^{i-1}\right)-H\left(Y_{i} \mid X_{1 i}, X_{2 i}\right)\right]+n \epsilon_{n} \\
& \leq \sum_{i=1}^{n}\left[H\left(Y_{i}\right)-H\left(Y_{i} \mid X_{1 i}, X_{2 i}\right)\right]+n \epsilon_{n} \\
& =\sum_{i=1}^{n} I\left(X_{1 i}, X_{2 i} ; Y_{i}\right)+n \epsilon_{n}
\end{array}
$$

We introduce a time-sharing random variable $Q[5, \mathrm{p}$. 397] as follows. Let $Q$ be uniformly distributed on $\{1, \ldots, n\}$ and be 
independent of $U^{n}, V^{n}$. Let the random variables $X_{1}$ and $X_{2}$ be such that

$$
\begin{aligned}
p\left(x_{1 i}, x_{2 i} \mid \mathbf{u}_{i}, \mathbf{v}_{i}, \mathbf{u}_{i}^{c}, \mathbf{v}_{i}^{c}\right) & \\
& =p\left(x_{1}, x_{2} \mid u^{m}, v^{m}, u_{m+1}^{n}, v_{m+1}^{n}, Q=i\right)
\end{aligned}
$$

where

$$
\begin{aligned}
& \mathbf{U}_{i}^{c} \triangleq U^{n} \backslash \mathbf{U}_{i} \\
& \mathbf{V}_{i}^{c} \triangleq V^{n} \backslash \mathbf{V}_{i}
\end{aligned}
$$

Then,

$$
\begin{aligned}
\sum_{i=1}^{n} I\left(X_{1 i} ; Y_{i} \mid X_{2 i}, \mathbf{V}_{i}\right) & =n I\left(X_{1} ; Y \mid X_{2}, V^{m}, Q\right) \\
\sum_{i=1}^{n} I\left(X_{2 i} ; Y_{i} \mid X_{1 i}, \mathbf{U}_{i}\right) & =n I\left(X_{2} ; Y \mid X_{1}, U^{m}, Q\right) \\
\sum_{i=1}^{n} I\left(X_{1 i}, X_{2 i} ; Y_{i}\right) & =n I\left(X_{1}, X_{2} ; Y \mid Q\right)
\end{aligned}
$$

Combining (26), (27) and (28) with (20), (21) and (22) completes the proof.

\section{A Finite-Letter OUter Bound}

It can be shown that the outer bound in Theorem 3 is equivalent to the following form

$$
\mathbf{H} \in \mathcal{R}(\mathcal{S}) \triangleq \operatorname{co}\left\{\bigcup_{\mathbf{p} \in \mathcal{S}} \mathcal{R}(\mathbf{p})\right\}
$$

where

$$
\begin{aligned}
\mathbf{H} & \triangleq[H(U \mid V), H(V \mid U), H(U, V)] \\
\mathbf{p} & \triangleq p\left(x_{1}, x_{2} \mid u^{m}, v^{m}\right) \\
\mathcal{S} & \triangleq\left\{\mathbf{p}: X_{1} \longrightarrow U^{n} \longrightarrow V^{n} \longrightarrow X_{2}\right\} \\
\mathcal{R}(\mathbf{p}) & \triangleq\left\{\left[R_{1}, R_{2}, R_{3}\right]:\left\{\begin{array}{l}
R_{1} \leq I\left(X_{1} ; Y \mid X_{2}, V^{m}\right) \\
R_{2} \leq I\left(X_{2} ; Y \mid X_{1}, U^{m}\right) \\
R_{3} \leq I\left(X_{1}, X_{2} ; Y\right)
\end{array}\right\}\right.
\end{aligned}
$$

and $\operatorname{co}\{\cdot\}$ represents the closure of the convex hull of the set argument.

Although the mutual information expressions in this outer bound, i.e., those in (33), depend only on the finiteletter conditional probability distribution of the channel inputs $\mathbf{p}=p\left(x_{1}, x_{2} \mid u^{m}, v^{m}\right)$, which involves only a finite number of source samples $\left(U^{m}, V^{m}\right)$, the constraint on $p\left(x_{1}, x_{2} \mid u^{m}, v^{m}\right), X_{1} \longrightarrow U^{n} \longrightarrow V^{n} \longrightarrow X_{2}$, is an $n$ letter constraint, which involves $n$ samples of the sources, $\left(U^{n}, V^{n}\right)$. Thus, we view his outer bound as an $n$-letter bound, which is incomputatble when $n$ goes to infinity. In the rest of this section, we will find a finite-letter necessary condition for this $n$-letter Markov chain constraint, or equivalently, we will find a set $\mathcal{S}^{\prime}$ for the set of admissible conditional probability distributions for the channel inputs $p\left(x_{1}, x_{2} \mid u^{m}, v^{m}\right)$ that satisfy

$$
\mathcal{S}^{\prime} \supseteq \mathcal{S}
$$

and, also can be characterized by finite-letter expressions.

We introduce our matrix notation for probability distributions [4]. For a pair of discrete random variables $X$ and $Y$, which take values in $\mathcal{X}=\left\{x_{1}, x_{2}, \ldots, x_{k}\right\}$ and $\mathcal{Y}=\left\{y_{1}, y_{2}, \ldots, y_{l}\right\}$, respectively, the joint distribution matrix $P_{X Y}$ is defined as

$$
P_{X Y}(i, j) \triangleq \operatorname{Pr}\left(X=x_{i}, Y=y_{j}\right)
$$

where $P_{X Y}(i, j)$ denotes the $(i, j)$-th element of the matrix $P_{X Y}$. The marginal distribution of a random variable $X$ is defined as a diagonal matrix with

$$
P_{X}(i, i) \triangleq \operatorname{Pr}\left(X=x_{i}\right)
$$

The vector-form marginal distribution $p_{X}$ is defined as

$$
p_{X}(i) \triangleq \operatorname{Pr}\left(X=x_{i}\right)
$$

or equivalently

$$
p_{X}=P_{X} \mathbf{e}
$$

where $\mathbf{e}$ is the vector of all ones. $p_{X}$ can also be defined as $p_{X} \triangleq P_{X Y}$ for some $Y$ where the size of the alphabet of $Y$, $|\mathcal{Y}|$, is equal to one. For conditional distributions, we define $P_{X Y \mid z}$ as

$$
P_{X Y \mid z}(i, j) \triangleq \operatorname{Pr}\left(X=x_{i}, Y=y_{j} \mid Z=z\right)
$$

A vector-form conditional distribution $p_{X \mid z}$ is defined as

$$
p_{X \mid z}(i) \triangleq \operatorname{Pr}\left(X=x_{i} \mid Z=z\right)
$$

or equivalently, $p_{X \mid z}(i) \triangleq P_{X Y \mid z}$ for some $Y$ where the size of the alphabet of $Y,|\mathcal{Y}|$, is equal to one.

We define a new quantity, $\tilde{P}_{X Y}$, which will play an important role in the rest of the paper, as

$$
\tilde{P}_{X Y} \triangleq P_{X}^{-\frac{1}{2}} P_{X Y} P_{Y}^{-\frac{1}{2}}
$$

Since $p_{X} \triangleq P_{X Y}$ for some $Y$ where the size of the alphabet of $Y,|\mathcal{Y}|$, is equal to one, we define

$$
\tilde{p}_{X}=P_{X}^{-\frac{1}{2}} P_{X Y} P_{Y}^{-\frac{1}{2}}=P_{X}^{-\frac{1}{2}} p_{X}
$$

The conditional distributions $\tilde{P}_{X Y \mid z}$ and $\tilde{p}_{X \mid y}$ can be defined similarly.

A joint distribution matrix, $P_{X Y}$, is a matrix whose entries are nonnegative and sum to 1 . Due to this constraint, not every matrix will qualify as a $\tilde{P}_{X Y}$ corresponding to a joint distribution matrix. A necessary and sufficient condition for $\tilde{P}_{X Y}$ to correspond to a joint distribution matrix is given in the following lemma.

Lemma 1 ([4]) An $m \times n$ non-negative matrix $P$ is a joint distribution matrix with marginal distributions $P_{X}$ and $P_{Y}$, i.e., $P \mathbf{e}=p_{X} \triangleq P_{X} \mathbf{e}$ and $P^{T} \mathbf{e}=p_{Y} \triangleq P_{Y} \mathbf{e}$, if and only if the singular value decomposition (SVD) of $\tilde{P} \triangleq P_{X}^{-\frac{1}{2}} P P_{Y}^{-\frac{1}{2}}$ satisfies

$$
\tilde{P}=U \Lambda V^{T}=p_{X}^{\frac{1}{2}}\left(p_{Y}^{\frac{1}{2}}\right)^{T}+\sum_{i=2}^{l} \lambda_{i} \mathbf{u}_{i} \mathbf{v}_{i}^{T}
$$


where $U \triangleq\left[\mathbf{u}_{1}, \ldots, \mathbf{u}_{l}\right]$ and $V \triangleq\left[\mathbf{v}_{1}, \ldots, \mathbf{v}_{l}\right]$ are two unitary matrices, $\Lambda \triangleq \operatorname{diag}\left[\lambda_{1}, \ldots, \lambda_{l}\right]$ and $l=\min (m, n) ; \mathbf{u}_{1}=p_{X}^{\frac{1}{2}}$, $\mathbf{v}_{1}=p_{Y}^{\frac{1}{2}}$, and $\lambda_{1}=1 \geq \lambda_{2} \geq \cdots \geq \lambda_{l} \geq 0$. That is, all of the singular values of $\tilde{P}$ are between 0 and 1 , the largest singular value of $\tilde{P}$ is 1 , and the corresponding left and right singular vectors are $p_{X}^{\frac{1}{2}}$ and $p_{Y}^{\frac{1}{2}}$.

This lemma implies that there is a one-to-one relationship between $P$ and $\tilde{P}$. It is easy to see from (41) that there is a unique $\tilde{P}$ for every $P$. Conversely, any given $\tilde{P}$ satisfying (43) gives a unique pair of marginal distributions $\left(P_{X}, P_{Y}\right)$, which is specified by the left and right singular vectors corresponding to its largest singular value. Then, from (41), using $\tilde{P}$ and $\left(P_{X}, P_{Y}\right)$ specified by its singular vectors, we obtain a unique $P$ as

$$
P=P_{X}^{\frac{1}{2}} \tilde{P} P_{Y}^{\frac{1}{2}}
$$

Because of this one-to-one relationship, exploring all possible joint distribution matrices $P$ is equivalent to exhausting all possible matrices $\tilde{P}$ satisfying (43).

In [4], we provided a new data processing inequality, a necessary condition for a Markov chain, as follows.

Lemma 2 ([4]) If $X \rightarrow Y \rightarrow Z$, then

$$
\lambda_{i}\left(\tilde{P}_{X Z}\right) \leq \lambda_{i}\left(\tilde{P}_{X Y}\right) \lambda_{2}\left(\tilde{P}_{Y Z}\right) \leq \lambda_{i}\left(\tilde{P}_{X Y}\right)
$$

where $i=2, \ldots, \operatorname{rank}\left(\tilde{P}_{X Z}\right)$, and $\lambda_{i}(\cdot)$ denotes the $i$-th largest singular value of a matrix.

We have also shown in [4] the following lemma, which provides a relation between $\lambda_{i}\left(\tilde{P}_{X^{n} Y^{n}}\right)$ and $\lambda_{2}\left(\tilde{P}_{X Y}\right)$.

Lemma 3 ([4]) For a pair of i.i.d. sequences $\left(X^{n}, Y^{n}\right)$ characterized by a joint distribution $P_{X Y}$, the ordered singular values of $\tilde{P}_{X^{n} Y^{n}}$ are

$$
\left\{1, \lambda_{2}\left(\tilde{P}_{X Y}\right), \ldots, \lambda_{2}\left(\tilde{P}_{X Y}\right), \ldots\right\}
$$

where the second through the $n+1$-st singular values are all equal to $\lambda_{2}\left(\tilde{P}_{X Y}\right)$.

Combining Lemma 2 and Lemma 3, we have the following lemma.

Lemma 4 Let $\left(U^{n}, V^{n}\right)$ be a pair of i.i.d. sequences of length $n$, and let the random variables $X_{1}, X_{2}$ satisfy $X_{1} \longrightarrow$ $U^{n} \longrightarrow V^{n} \longrightarrow X_{2}$. Let us define $\mathbf{U}$ as an arbitrary subset of $U^{n}$, i.e.,

$$
\mathbf{U} \triangleq\left\{U_{i_{1}}, \ldots, U_{i_{l}}\right\} \subset U^{n}
$$

and similarly,

$$
\mathbf{V} \triangleq\left\{V_{j_{1}}, \ldots, V_{j_{k}}\right\} \subset V^{n}
$$

Then, for $i=2, \ldots, \operatorname{rank}\left(\tilde{P}_{X_{1} X_{2}}\right)$,

$$
\lambda_{i}\left(\tilde{P}_{X_{1} X_{2} \mid \mathbf{u v}}\right) \leq \lambda_{2}\left(\tilde{P}_{U V}\right)
$$

where $\lambda_{i}(\cdot)$ denotes the $i$-th largest singular value of a matrix.
Proof: We consider a special case of $(\mathbf{U}, \mathbf{V})$ as follows. We define $\mathbf{U} \triangleq\left\{U_{1}, \ldots, U_{l}\right\}$ and $\mathbf{V} \triangleq$ $\left\{V_{1}, \ldots, V_{m}, V_{l+1}, \ldots, V_{l+k-m}\right\}$. We also define the complements of these two sequence as $\mathbf{U}^{c} \triangleq U^{n} \backslash \mathbf{U}$ and $\mathbf{V}^{c} \triangleq$ $V^{n} \backslash \mathbf{V}$. If $\mathbf{U}$ and $\mathbf{V}$ take other forms, we can transform them to the form we defined above by permutation. Then,

$$
\tilde{P}_{X_{1} X_{2} \mid \mathbf{u v}}=\tilde{P}_{X_{1} \mathbf{U}^{c} \mid \mathbf{u}} \tilde{P}_{\mathbf{U}^{c} \mathbf{V}^{c} \mid \mathbf{u v}} \tilde{P}_{\mathbf{V}^{c} X_{2} \mid \mathbf{v}}
$$

Furthermore,

$$
\begin{aligned}
\tilde{P}_{\mathbf{U}^{c} \mathbf{V}^{c} \mid \mathbf{u v}}= & \tilde{p}_{V_{m+1}^{l} \mid u_{m+1}^{l}} \otimes \tilde{p}_{U_{l+1}^{l+k-m} \mid v_{l+1}^{l+k-m}} \otimes \\
& \otimes \tilde{P}_{U_{l+k-m+1}^{n}} V_{l+k-m+1}^{n}
\end{aligned}
$$

As mentioned earlier, a vector marginal distribution can be viewed as a joint distribution matrix with a random variable whose size of the alphabet is equal to 1 . Since the rank of a vector is 1 , from Lemma 1 , the only singular value of $\tilde{p}_{V_{m+1}^{l} \mid u_{m+1}^{l}}$ (and of $\tilde{p}_{U_{l+1}^{l+k-m} \mid v_{l+1}^{l+k-m}}$ ) is equal to 1 . Then,

$$
\lambda_{i}\left(\tilde{P}_{\mathbf{U}^{c} \mathbf{V}^{c} \mid \mathbf{u v}}\right)=\lambda_{i}\left(\tilde{P}_{U_{l+k-m+1}^{n} V_{l+k-m+1}^{n}}\right)
$$

Combining (45), (49), and (51), we obtain

$$
\lambda_{i}\left(\tilde{P}_{X_{1} X_{2} \mid \mathbf{u v}}\right) \leq \lambda_{2}\left(\tilde{P}_{U V}\right)
$$

which completes the proof.

Lemma 4 provides a finite-letter necessary condition for the $n$-letter Markov chain $X_{1} \longrightarrow U^{n} \longrightarrow V^{n} \longrightarrow X_{2}$. This means that, if a conditional probability distribution $p\left(x_{1}, x_{2} \mid u^{m}, v^{m}\right)$ satisfies the constraint $X_{1} \longrightarrow U^{n} \longrightarrow$ $V^{n} \longrightarrow X_{2}$, then it satisfies (48) as well. In other words, $\mathcal{S}$, the set that contains all $p\left(x_{1}, x_{2} \mid u^{m}, v^{m}\right)$ satisfying $X_{1} \longrightarrow$ $U^{n} \longrightarrow V^{n} \longrightarrow X_{2}$, is a subset of the set that contains all $p\left(x_{1}, x_{2} \mid u^{m}, v^{m}\right)$ satisfying (48). That is, if we define

$$
\mathcal{S}^{\prime} \triangleq \bigcap_{\mathbf{U} \subseteq U^{m}, \mathbf{V} \subseteq V^{m}} \mathcal{S}_{\mathbf{u v}}
$$

where

$$
\mathcal{S}_{\mathbf{u v}} \triangleq\left\{p\left(x_{1}, x_{2} \mid u^{m}, v^{m}\right): \lambda_{i}\left(\tilde{P}_{X_{1} X_{2} \mid \mathbf{U V}}\right) \leq \lambda_{2}\left(\tilde{P}_{U V}\right)\right\}
$$

then,

$$
\mathcal{S} \subseteq \mathcal{S}^{\prime}
$$

A direct consequence of the set relation in (55) is

$$
\mathcal{R}(\mathcal{S}) \subseteq \mathcal{R}\left(\mathcal{S}^{\prime}\right)
$$

From the above discussion, we obtain the main result of our paper, which is stated in the following theorem.

Theorem 4 If a pair of i.i.d. sources $(U, V)$ with joint distribution $p(u, v)$ can be transmitted reliably through a discrete, memoryless, multiple access channel characterized by $p\left(y \mid x_{1}, x_{2}\right)$, then

$$
\begin{aligned}
& H(U \mid V) \leq I\left(X_{1} ; Y \mid X_{2}, V^{m}, Q\right) \\
& H(V \mid U) \leq I\left(X_{2} ; Y \mid X_{1}, U^{m}, Q\right) \\
& H(U, V) \leq I\left(X_{1}, X_{2} ; Y \mid Q\right)
\end{aligned}
$$


where $\left(U^{m}, V^{m}\right)$ are $m$ samples of the i.i.d. sources, for some finite non-negative integer $m$, random variable $Q$ independent of $\left(U^{m}, V^{m}\right)$, and for random variables $X_{1}, X_{2}$, $p\left(x_{1}, x_{2} \mid u^{m}, v^{m}\right)$ such that, for any $\mathbf{U} \subseteq U^{m}$ and $\mathbf{V} \subseteq V^{m}$,

$$
\lambda_{i}\left(\tilde{P}_{X_{1} X_{2} \mid \mathbf{u v} q}\right) \leq \lambda_{2}\left(\tilde{P}_{U V}\right)
$$

Equivalently,

$$
\mathbf{H} \in \mathcal{R}\left(\mathcal{S}^{\prime}\right)=\operatorname{co}\left\{\bigcup_{\mathbf{p} \in \mathcal{S}^{\prime}} \mathcal{R}(\mathbf{p})\right\}
$$

where

$$
\mathcal{S}^{\prime} \triangleq \bigcap_{\mathbf{U} \subseteq U^{m}, \mathbf{V} \subseteq V^{m}} \mathcal{S}_{\mathbf{u v}}
$$

and

$$
\mathcal{S}_{\mathbf{u v}} \triangleq\left\{p\left(x_{1}, x_{2} \mid u^{m}, v^{m}\right): \lambda_{i}\left(\tilde{P}_{X_{1} X_{2} \mid \mathbf{U V}}\right) \leq \lambda_{2}\left(\tilde{P}_{U V}\right)\right\}
$$

This outer bound is a finite-letter bound because the constraints on $p\left(x_{1}, x_{2} \mid u^{m}, v^{m}\right)$ in (60) involve no more than $m$ samples of the sources, where $m$ is a finite integer. From the derivation in (20), we know that the outer bound is tighter when $m$ is larger. However, when $m$ is larger, there are more choices for $\mathbf{U}$ and $\mathbf{V}$ in (62). Thus, there exists a tradeoff between the tightness of the bound and the complexity of evaluating the bound.

\section{Discussion, $m=1$}

In this section, we will specialize our results for $m=1$. When $m=1$,

$$
\mathcal{S}=\left\{p\left(x_{1}, x_{2} \mid u_{1}, v_{1}\right): X_{1} \longrightarrow U^{n} \longrightarrow V^{n} \longrightarrow X_{2}\right\}
$$

and

$$
\mathcal{S}^{\prime}=\mathcal{S}_{\emptyset} \cap \mathcal{S}_{U_{1}} \cap \mathcal{S}_{V_{1}} \cap \mathcal{S}_{U_{1} V_{1}}
$$

where

$$
\begin{gathered}
\mathcal{S}_{\emptyset} \triangleq\left\{p\left(x_{1}, x_{2} \mid u_{1}, v_{1}\right): \lambda_{i}\left(\tilde{P}_{X_{1} X_{2}}\right) \leq \lambda_{2}\left(\tilde{P}_{U V}\right)\right\} \\
\mathcal{S}_{U_{1}} \triangleq\left\{p\left(x_{1}, x_{2} \mid u_{1}, v_{1}\right): \lambda_{i}\left(\tilde{P}_{X_{1} X_{2} \mid u_{1}}\right) \leq \lambda_{2}\left(\tilde{P}_{U V}\right)\right\} \\
\mathcal{S}_{V_{1}} \triangleq\left\{p\left(x_{1}, x_{2} \mid u_{1}, v_{1}\right): \lambda_{i}\left(\tilde{P}_{X_{1} X_{2} \mid v_{1}}\right) \leq \lambda_{2}\left(\tilde{P}_{U V}\right)\right\} \\
\mathcal{S}_{U_{1} V_{1}} \triangleq\left\{p\left(x_{1}, x_{2} \mid u_{1}, v_{1}\right): \lambda_{i}\left(\tilde{P}_{X_{1} X_{2} \mid u_{1} v_{1}}\right) \leq \lambda_{2}\left(\tilde{P}_{U V}\right)\right\}
\end{gathered}
$$

From this example, we see that there is only one constraint on $p\left(x_{1}, x_{2} \mid u_{1}, v_{1}\right)$ for the set $\mathcal{S}$, that is the Markov chain constraint $X_{1} \longrightarrow U^{n} \longrightarrow V^{n} \longrightarrow X_{2}$. However, Lemma 4 shows that the necessary condition for $X_{1} \longrightarrow U^{n} \longrightarrow$ $V^{n} \longrightarrow X_{2}$ involves not only $p\left(x_{1}, x_{2} \mid u_{1}, v_{1}\right)$, but also $p\left(x_{1}, x_{2} \mid u_{1}\right), p\left(x_{1}, x_{2} \mid v_{1}\right)$, and $p\left(x_{1}, x_{2}\right)$, as shown in (66) through (69). This leads to a set of multiple constraints on $p\left(x_{1}, x_{2} \mid u_{1}, v_{1}\right)$ for the set $\mathcal{S}^{\prime}$, because $p\left(x_{1}, x_{2} \mid u_{1}\right)$, $p\left(x_{1}, x_{2} \mid v_{1}\right)$, and $p\left(x_{1}, x_{2}\right)$ can all be written as linear combinations of $p\left(x_{1}, x_{2} \mid u_{1}, v_{1}\right)$.

We observe that when $m=1$, the expressions in Theorem 4, i.e., (57), (58), and (59), agree with those in the achievability scheme of Cover, El Gamal and Salehi without common information between the sources, i.e., (7), (8), and (9). Thus, the gap between the achievablity scheme of Cover, El Gamal and Salehi, and the converse in this paper results from the fact that the feasible sets for the conditional probability distribution $\mathbf{p}=p\left(x_{1}, x_{2} \mid u, v\right)$ are different. In the achievability scheme of Cover, El Gamal and Salehi, $\mathbf{p}$ belongs to

$$
\mathcal{S}^{\prime \prime} \triangleq\left\{p\left(x_{1}, x_{2} \mid u, v\right): p\left(x_{1}, x_{2} \mid u, v\right)=p\left(x_{1} \mid u\right) p\left(x_{2} \mid v\right)\right\}
$$

since for the achievability, we need $X_{1} \longrightarrow U \longrightarrow V \longrightarrow X_{2}$, as stated in (10). Whereas, in our converse, $\mathbf{p} \in \mathcal{S} \subseteq \mathcal{S}^{\prime}$. It is easy to see that

$$
\mathcal{S}^{\prime \prime} \subseteq \mathcal{S} \subseteq \mathcal{S}^{\prime}
$$

Therefore, when $m=1$, even though the mutual information expressions in the achievability and the converse are the same, their actual values will be different, since they will be evaluated using the conditional probability distributions that belong to different feasible sets.

\section{CONCLUSION}

The capacity region of the multiple access channel with correlated sources is an open problem. Cover, El Gamal and Salehi gave a single-letter achievable region, and also a converse in terms of some incomputable $n$-letter mutual informations. In this paper, we proposed an outer bound for the capacity region of the multiple access channel with correlated sources in finite-letter expressions, by utilizing a new necessary condition for the Markov chain constraint on the valid channel input distributions.

\section{REFERENCES}

[1] T. M. Cover, A. El Gamal, and M. Salehi, "Multiple access channel with arbitrarily correlated sources," IEEE Trans. Inform. Theory, vol. 26, pp. 648-657, Nov. 1980

[2] H. S. Witsenhausen, "On sequences of pairs of dependent random variables," SIAM Journal on Applied Mathematics, vol. 28, pp. 100-113, Jan. 1975.

[3] G. Dueck, "A note on the multiple access channel with correlated sources," IEEE Trans. Inform. Theory, vol. 27, pp. 232-235, Mar. 1981.

[4] W. Kang and S. Ulukus, "A single-letter upper bound for the sum rate of multiple access channels with correlated sources," in Proc. 39th Asilomar Conf. on Signals, Systems and Computers, Pacific Grove, CA, Oct. 2005.

[5] T. M. Cover and J. A. Thomas, Elements of Information Theory. John Wiley and Sons, 1991. 